\title{
Operational and Dosimetric Aspects of Pediatric PET/CT
}

\author{
Frederic H. Fahey ${ }^{1,2}$, Alison Goodkind ${ }^{1}$, Robert D. MacDougall ${ }^{1}$, Leah Oberg ${ }^{1}$, Sonja I. Ziniel ${ }^{3}$, Richard Cappock $^{1}$, \\ Michael J. Callahan ${ }^{1,2}$, Neha Kwatra ${ }^{1,2}$, S. Ted Treves ${ }^{2,4}$, and Stephan D. Voss ${ }^{1,2}$ \\ ${ }^{I}$ Division of Nuclear Medicine and Molecular Imaging, Department of Radiology, Boston Children's Hospital, Boston, \\ Massachusetts; ${ }^{2}$ Department of Radiology, Harvard Medical School, Boston, Massachusetts; ${ }^{3}$ Section of Pediatric Hospital Medicine, \\ Department of Pediatrics, University of Colorado School of Medicine and Children's Hospital Colorado, Aurora, Colorado; and \\ ${ }^{4}$ Department of Radiology, Brigham and Women's Hospital, Boston, Massachusetts
}

Learning Objectives: On successful completion of this activity, participants should be able to (1) list 3 factors that affect CT radiation dose; (2) discuss 3 ways that CT is used in the context of PET/CT; and (3) describe various approaches to integrating PET/CT and diagnostic CT in children.

Financial Disclosure: Dr. Fahey is a board member of the American Association of Physicists in Medicine and an employee of Boston Children's Hospital. The authors of this article have indicated no other relevant relationships that could be perceived as a real or apparent conflict of interest.

CME Credit: SNMMI is accredited by the Accreditation Council for Continuing Medical Education (ACCME) to sponsor continuing education for physicians. SNMMI designates each JNM continuing education article for a maximum of 2.0 AMA PRA Category 1 Credits. Physicians should claim only credit commensurate with the extent of their participation in the activity. For CE credit, SAM, and other credit types, participants can access this activity through the SNMMI website (http://www.snmmilearningcenter.org) through September 2020.

No consistent guidelines exist for the acquisition of a CT scan as part of pediatric PET/CT. Given that children may be more vulnerable to the effects of ionizing radiation, it is necessary to develop methods that provide diagnostic-quality imaging when needed, in the shortest time and with the lowest patient radiation exposure. This article describes the basics of CT dosimetry and $\mathrm{PET} / \mathrm{CT}$ acquisition in children. We describe the variability in pediatric PET/CT techniques, based on a survey of $19 \mathrm{PET} / \mathrm{CT}$ pediatric institutions in North America. The results of the survey demonstrated that, although most institutions used automatic tube current modulation, there remained a large variation of practice, on the order of a factor of 2-3, across sites, pointing to the need for guidelines. We introduce the approach developed at our institution for using a multiseries PET/CT acquisition technique that combines diagnostic-quality CT in the essential portion of the field of view and a low-dose technique to image the remainder of the body. This approach leads to a reduction in radiation dose to the patient while combining the PET and the diagnostic CT into a single acquisition. The standardization of pediatric PET/CT provides an opportunity for a reduction in the radiation dose to these patients while maintaining an appropriate level of diagnostic image quality.

Key Words: pediatric; dosimetry; PET/CT; computed tomography

J Nucl Med 2017; 58:1360-1366

DOI: 10.2967/jnumed.116.182899

H ybrid imaging using PET/CT provides crucial information in the diagnosis and management of a variety of pediatric conditions including oncology, neurology, and cardiology (1). The use of PET/ $\mathrm{CT}$ has transformed both staging and tumor response assessments in

Received Mar. 16, 2017; revision accepted Jul. 5, 2017

For correspondence or reprints contact: Frederic $\mathrm{H}$. Fahey, Division of Nuclear Medicine and Molecular Imaging, Boston Children's Hospital, 300 Longwood Ave., Boston, MA 02115.

E-mail: frederic.fahey@childrens.harvard.edu

Published online Jul. 7, 2017.

COPYRIGHT (c) 2017 by the Society of Nuclear Medicine and Molecular Imaging. several pediatric malignancies, including Hodgkin and non-Hodgkin lymphoma, Ewing sarcoma, and Langerhans cell histiocytosis (2-7).

There continues to be concern regarding the radiation dose associated with medical imaging, particularly CT (8-15), although the relationship between exposure and radiation-related illness remains the subject of considerable debate (16). Although children typically receive less administered activity from nuclear medicine procedures than adults, they are considered to be particularly sensitive to potential carcinogenic effects of ionizing radiation. This is due to both the enhanced sensitivity of their rapidly dividing cells and the longer lifetimes over which negative outcomes potentially could be manifest. In the Biologic Effects of Ionizing Radiation (BEIR) VII phase 2 report from the National Academy of Sciences, the carcinogenic risk for a 10-y-old was estimated to be twice that of a 40-y-old adult; and a 1-y-old, 3 times that level (17). These potential risks vary across tumor type, and young girls under the age of $20 \mathrm{y}$ are considered to be at higher risk than boys, primarily due to breast cancer risk.

Although minimizing the radiation dose to children may be desirable, it is essential that this not come at the expense of jeopardizing the quality of the clinical information necessary for the patient's care. Any potential risk also must be considered in the overall context of the patient's care, particularly in the case of oncology. Given these concerns, it remains prudent for practitioners to eliminate unnecessary radiation exposure by optimizing acquisition parameters to use the minimum dose while maintaining high diagnostic (Dx) quality $(16,18-20)$. For PET/CT, the radiation dose must be considered from both the radiopharmaceutical and the CT scan $(18,21)$. There is also a desire to reduce the duration of sedation or anesthesia by eliminating duplicate CT in children undergoing both Dx CT and PET/CT. The optimization of the PET/CT acquisition will lead to safer, more efficient PET/CT while maintaining its high clinical value.

This article reviews the basics of CT dosimetry in the context of pediatric PET/CT. The state of the practice of PET/CT in children, as revealed by a survey of dedicated pediatric institutions in North America, will be discussed. Lastly, an optimized approach to pediatric PET/CT will be presented. 


\section{BASICS OF CT DOSIMETRY}

CT provides a map of the differential absorption of x-rays passing through the patient, resulting in a $3 \mathrm{D}$ representation of tissue density (22). This map depends on both electron density and the average number of protons per atom (i.e., the effective $\mathrm{Z}$ number) of the material. Electrons liberated from the cathode by thermionic emission are accelerated across the CT x-ray tube through an applied voltage where they strike the anode and deaccelerate, leading to the production of bremsstrahlung and characteristic $\mathrm{x}$-rays. The tube current in milliamperes $(\mathrm{mA})$ characterizes the number of electrons striking the anode per second. The number of electrons striking the anode (and subsequently the number of $\mathrm{x}$-rays generated) is directly related to the product of the tube current and the exposure duration (in $\mathrm{mAs}$ ). Thus, the patients' radiation dose from $\mathrm{x}$-rays is directly proportional to $\mathrm{mAs}$.

The peak voltage across the tube (in $\mathrm{kVp}$ ) defines the energy of the electrons striking the anode (in $\mathrm{keV}$ ) and thus the energy of the resultant bremsstrahlung X-rays. Higher-energy electrons are more efficient at generating $\mathrm{x}$-rays, and these are more likely to exit the tube and reach the patient. The radiation dose delivered to the patient depends on several factors; however, the dose varies roughly as the square of the tube voltage.

As the CT x-ray tube rotates around the patient, some fraction of the emitted x-rays passes through the patient and subsequently is detected by a $2 \mathrm{D}$ array of radiation detectors. The $\mathrm{x}$-ray tube and the detector array are in a fixed geometry rotating about the patient, typically with a 0.33 - to 1 -s rotation time. The acquired projection data can be reconstructed into a 3D series of parallel planes. During CT acquisition, the patient bed moves continuously, allowing a volume of the patient to be imaged in a helical fashion. The table speed normalized by the collimated beam width at isocenter is reported as the pitch given by

$$
\text { Pitch }=\frac{\text { (table distance traveled in a single gantry rotation) }}{\text { (width of the beam collimation) }} .
$$

When the table travels a distance equal to the beam width during a single gantry rotation, this corresponds to a pitch of unity (1:1). Higher pitch values (e.g., 1.5:1) derive from faster table speeds and may yield slightly under-sampled data, but result in lower radiation exposure or less scan time. Interpolation between the acquired axial data of higher pitch studies typically leads to an adequate reconstruction.

The combination of tube current $(\mathrm{mA})$, rotation time (s), and pitch define the effective mAs given by

$$
\text { Effective } \mathrm{mAs}=\frac{\mathrm{mA} \times \text { rotation time }(\mathrm{s})}{\text { pitch }} .
$$

Quantification of the CT signal is given in CT or Hounsfield units, which parameterize the mean linear attenuation coefficient within a voxel relative to that of water.

$$
\text { CT number (or Hounsfield units })=1,000 x \frac{\left(\mu_{V}-\mu_{\text {water }}\right)}{\mu_{\text {water }}},
$$

where $\mu_{V}$ is the measured mean linear attenuation coefficient within a voxel and $\mu_{\text {water }}$ is the linear attenuation coefficient for water given the $\mathrm{x}$-ray spectrum of the $\mathrm{CT}$ acquisition.

Automatic exposure control (AEC) - that is, automatic exposure adaptation to patient size and anatomic attenuation-is available on all state-of-the-art CT scanners, including those incorporated into
PET/CT systems. There are 2 aspects of AEC: automatic kV selection, currently available from one manufacturer (Siemens Healthineers), and automated tube current modulation (ATCM). A low-dose projection image (i.e., a "scout" or "topogram") provides a 2D attenuation map used for both $\mathrm{kV}$ selection and ATCM. The automatic $\mathrm{kV}$ selection optimizes the contrast-to-noise ratio for a given patient size and imaging task. ATCM achieves a similar level of image noise at each position of the scan. For instance, less radiation is necessary for imaging the lungs than the abdomen, and less is needed when scanning the anterior-posterior direction than the orthogonal lateral direction. The $\mathrm{mA}$ is modified according to user-defined parameters regarding a nominal image quality, that is, the Quality Reference mAs (QRM) for Siemens or the Noise Index (NI) for GE Healthcare (GE). Depending on the size and shape of the patient and the CT procedure being performed, AEC can yield a $25 \%-40 \%$ reduction in radiation dose to the patient (example shown in Fig. 1).

There are several dosimetric parameters used with CT $(21,22)$. The CT dose index (CTDI) characterizes the radiation dose delivered (in mGy) to a standard head or adult body phantom (16- or $32-\mathrm{cm}$ diameter, respectively) for a particular set of acquisition parameters. $\mathrm{CTDI}_{\mathrm{vol}}$ is the weighted average of CTDI values in the phantom center and on the periphery normalized by the pitch. The dose-length product (DLP, in $\mathrm{mGy}-\mathrm{cm}$ ) is the product of CTDI $\mathrm{vol}_{\mathrm{v}}$ and the axial length of the scan. CTDI ${ }_{\mathrm{vol}}$ and DLP are routinely displayed on the CT operator's console during an acquisition and recorded in the radiation dose structured report and DICOM header. $\mathrm{CTDI}_{\mathrm{vol}}$ and DLP do not represent the radiation dose to a particular patient but to a standard phantom. However, the $\mathrm{CTDI}_{\mathrm{vol}}$ can be modified for smaller or larger patients and reported as the size-specific dose estimate $(23,24)$.

\section{ACQUISITION OF PET/CT}

In PET/CT, the CT can be used for any of 3 distinct purposesattenuation correction, anatomic colocalization, or Dx interpretationor for a combination of these $(21,25)$. Attenuation correction accounts for differences in the location of positron annihilation events and the degree to which tissues "attenuate" the PET annihilation photons. Photons emitted from the center of the patient are less likely to be detected than those from the periphery. CT data can be incorporated into the reconstruction process for attenuation correction.

CT acquisition parameters and the resultant patient radiation dose can vary significantly depending on how the CT is to be used. When used only for attenuation correction, the CT dose can be substantially reduced while still providing adequate attenuation correction $(25,26)$. In this case, anatomic detail

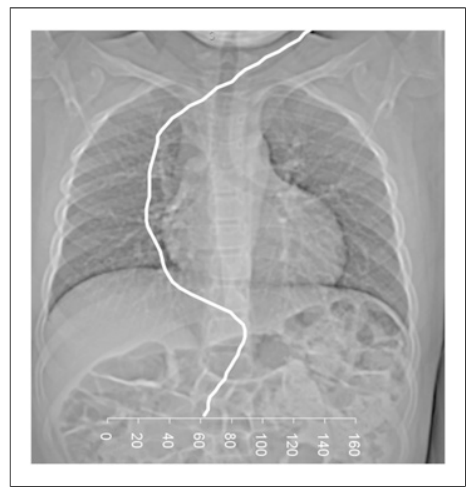

FIGURE 1. Example of AEC. The underlying image is a CT topogram of a chest CT. The white profile indicates the $m A s$ as a function of $z$-axis position. The $m A s$ is higher through the shoulders due to the increase in transverse dimension and attenuating structures (bone), then drops significantly over the lungs and rises again over the abdomen when the more attenuating solid organ viscera are encountered. 
may be sufficiently compromised as to preclude accurate anatomic colocalization. When the CT is used for anatomic correlation of the PET data, the CT need not be of Dx image quality but adequate to discern the anatomic features of interest on PET. Finally, a Dx CT of the region of interest may be acquired. If the patient requires both Dx CT and PET, the option of acquiring both in a single imaging session not only is convenient and efficient for the patient but also provides an opportunity for dose reduction by potentially eliminating the need for an additional CT scan. For small children, it may also reduce the need for sedation to complete both scans. In many instances, the CT may be used for several purposes; for example, Dx CT may be used for anatomic correlation and attenuation correction, or CT for anatomic correlation may be used for attenuation correction.

Adjustments must be made to apply CT-based attenuation correction given the differences between the CT and PET data. The CT tube voltage is typically between 80 and $140 \mathrm{kVp}$ (effective photon energies between 40 and $80 \mathrm{keV}$ ) whereas the annihilation photon energy is $511 \mathrm{keV}$. A multilinear transformation between the reconstructed CT number and the linear attenuation coefficient for tissue at $511 \mathrm{keV}$ can adjust for this energy difference (27). An adjustment must also be made for the variation in spatial resolution between the two modalities (around $1 \mathrm{~mm}$ for CT compared with 5-10 $\mathrm{mm}$ for PET) to avoid artifacts at the boundary of objects. The CT data are blurred to the resolution of PET before applying the energy transformation. Thus, CT-based attenuation correction is applied as follows: acquire and reconstruct the CT scan, smooth to the resolution of PET, apply the energy transformation, reproject the transformed data to generate a correction matrix, and apply the correction during PET reconstruction.

For CT used for anatomic correlation, the image quality need only be adequate for this purpose. Thus, the mAs can be reduced by a factor of possibly 3-5, depending on the task at hand and the size of the patient. A Dx CT scan may be acquired in conjunction with the PET as it may increase the confidence with which a PET finding is correlated anatomically. For example, in abdominal viscera, lesion conspicuity is usually dependent on CT technique and properly timed contrast administration. Combining Dx CT and PET can improve efficiency, reduce examination time and possibly the requirement for sedation, and can be more convenient for the patient. When the Dx CT and PET cover the same region of the body (usually torso), the Dx CT can also be used for attenuation correction. However, when the Dx CT is applied to a specific region of the body (e.g., head/neck or chest) and whole-body PET is required, alternative strategies are needed as discussed below.

\section{PRACTICE OF PEDIATRIC PET/CT}

\section{Survey of North American Pediatric PET/CT Clinics}

Due to the lack of practice standards, we hypothesized that the acquisition of pediatric PET/CT may vary substantially from clinic to clinic. We sought to characterize the practice of pediatric PET/CT by performing a survey of 19 North American institutions: 14 dedicated pediatric hospitals and 5 large academic hospitals with significant pediatric practices (Table 1). Internal Review Board review was not required since no data from individual patients were requested, but rather standard institutional protocols for acquiring PET/CT were requested.

Survey data were collected and managed using REDCap (Research Electronic Data Capture) electronic data capture tools hosted at the University of Colorado School of Medicine (28). The
TABLE 1

Institutions Completing the Pediatric PET/CT Survey

Boston Children's Hospital

Texas Children's Hospital

St. Jude Children's Research Hospital

Cincinnati Children's Hospital Medical Center

University of Michigan CS Mott Children's Hospital

Children's Hospital Los Angeles

Lucile Packard Children's Hospital Stanford

Barnes-Jewish and Saint Louis Children's Hospitals

British Columbia Children's Hospital

Duke University

Children's Hospital of Pittsburgh

Children's Mercy Kansas City

Children's National Health System

Children's Hospital Colorado

The Hospital for Sick Children

Seattle Children's Hospital

Massachusetts General Hospital

Ann \& Robert H. Lurie Children's Hospital of Chicago

Children's Hospital of Philadelphia

survey was divided into 3 sections. First, we asked sites to provide general institutional information regarding the number of pediatric PET/CT studies performed per week and the manufacturer and model of the PET/CT scanner primarily used for pediatric imaging. Second, we inquired as to whether they acquired non-Dx (for attenuation correction or anatomic correlation) or Dx CT as part of PET/CT at their institution. Finally, we asked how they would acquire both non-Dx and Dx PET/CT on 3 hypothetical patients weighing 25,50 , and $75 \mathrm{~kg}$.

All 19 sites completed the survey within $3 \mathrm{wk}$. The number of pediatric PET/CT scans performed per week varied greatly, with median and mean values of 4.0 and 6.2 scans/wk, respectively (range of 1.5-20 scans/wk). Nine of 19 sites had scanners manufactured by GE, 5 by Siemens, and 5 by Philips. Only 2 of the 19 sites used intravenous CT contrast material at the time of PET/CT. Eighteen and 15 of 19 sites performed non-Dx CT and Dx CT scans in conjunction with pediatric PET/CT, respectively. Of those 15 performing Dx CT, 4 sites performed the Dx CT over the entire PET field of view, 6 performed the Dx CT over a limited region at the time of PET/CT, and 5 performed limited CT separate from the $\mathrm{PET} / \mathrm{CT}$ on the same scanner but at a different time.

In our comparison of the technical factors used by the sites, we limited our analysis to Siemens and GE scanners. In general, ATCM was used in the majority of both Dx and non-Dx protocols. When automatic $\mathrm{kV}$ selection was available, it was used primarily in Dx scans and only in one non-Dx protocol. This is reasonable since the primary reason for $\mathrm{kV}$ modification is to enhance visualization of contrast material, which is not relevant for non-Dx CT. Looking at the ATCM parameters, there was a high degree of variation between sites (Table 2). On Siemens scanners using CARE Dose 4D, QRM varied from 60 to 150 (factor of $2.5 \times$ ) for the $20-\mathrm{kg}$ patient and from 120 to $220(1.8 \times)$ for the $75-\mathrm{kg}$ patient for Dx examinations. For GE scanners, NI varied from 5 to 18 
TABLE 2

ATCM Parameters for GE NI Range and Siemens QRM Range

\begin{tabular}{|c|c|c|c|c|}
\hline \multirow[b]{2}{*}{ Patient weight (kg) } & \multicolumn{2}{|c|}{$\mathrm{NI}$ range } & \multicolumn{2}{|c|}{ QRM range (mAs) } \\
\hline & Dx & Non-Dx & Dx & Non-Dx \\
\hline 20 & $5-18(12.97)$ & $20-30(28.5)$ & $60-150(140)$ & $35-80^{*}$ \\
\hline 50 & $10-18(13.5)$ & $20-40(29)$ & $120-180$ (150) & $35-80^{*}$ \\
\hline 75 & $11-18(15)$ & 20-40 (29) & $120-220(150)$ & $35-80^{*}$ \\
\hline
\end{tabular}

(3.6x) for the $20-\mathrm{kg}$ patient and from 11 to $18(1.6 \times)$ for the $75-\mathrm{kg}$ patient for Dx studies.

\section{Boston Children's Hospital Approach}

Traditionally, the CT component of a torso PET/CT examination has been performed at our institution as a low-dose non-Dx scan. If indicated, this has been followed by Dx CT limited to the anatomic area of clinical concern. We recognized that we could reduce imaging time and radiation dose to the patient by integrating the non-Dx and the Dx CT data, eliminating duplicate scanning over regions where the Dx CT was acquired. The Dx CT provided more than sufficient image data for anatomic correlation and could also be used for attenuation correction. However, previous software versions for our scanner did not permit merging separately acquired CT series into a single reconstructed non-Dx acquisition or acquiring a multiseries CT scan that incorporated both the non-Dx and the Dx CT into a single acquisition. Therefore, we collaborated with the manufacturer (Siemens Healthineers) to develop the capability for acquiring multiseries CT where the patient receives only the dose required for each area, with minimal overlap. For example, a non-Dx scan may be acquired over the head, neck, and thoracic regions followed by a Dx scan over the abdomen and then a non-Dx scan over the pelvis to the midthighs. These 3 segments are then merged into a single scan used for attenuation correction, anatomic correlation, and Dx CT without incident. Once feasibility was established, a multiseries CT acquisition was implemented.

We hypothesized that AEC could provide reasonable $\mathrm{CT}$ acquisition parameters for children of varying size. Thus, a phantom experiment was performed using our standard clinical CT protocols. All data were acquired using a Siemens mCT 40 PET/CT scanner using CARE KV and CARE Dose 4D for both the non-Dx and the Dx CT. At our institution, we use different protocols for patients under and over $55 \mathrm{~kg}$ for both non-Dx and Dx CT scans. For patients under $55 \mathrm{~kg}$, our Dx CT protocol uses CARE Dose 4D, a QRM of 150 , strength set to weak, CARE kV "on"

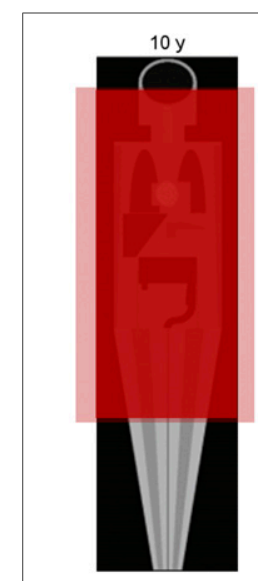

Scenario 1: Whole body $\mathrm{Dx}+$ Whole body AC (reference $\mathrm{kV}$ set to 120), and the Dose Optimizer set to 7. For the non-Dx CT, all parameters were as above except a QRM of 20 and 35 was used for patients under and over $55 \mathrm{~kg}$, respectively.

We imaged anthropomorphic, tissue-equivalent CT phantoms (CIRS, model 007TE) for 5 different sizes of patient (adult patient and 15-, 10-, 5-, and 1-y-old patients). For each age, there were separate phantoms for the head, thorax, and abdomen. $\mathrm{CTDI}_{\mathrm{vol}}(32-\mathrm{cm}$ body, 16-cm head) was recorded for each acquisition and multiplied by a nominal scan length for each region based on age, using conversion factors reported by Deak et al. (29) to calculate the DLP.

Effective dose (ED) was estimated for each region using DLP conversion factors based on International Council on Radiation Protection Report 103 (29). Five PET/CT imaging scenarios were considered (Fig. 2): (1) torso Dx followed by torso non-Dx, (2) torso Dx only, (3) torso non-Dx only, (4) abdominal Dx (from just above the diaphragm to just above the pelvis) followed by torso non-Dx, and (5) multiseries with non-Dx head and chest plus

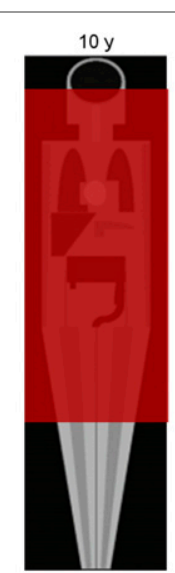

Scenario 2: Whole Body Dx only

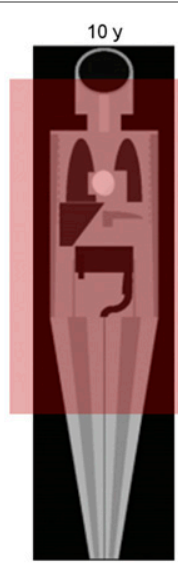

Scenario 3: Whole Body AC

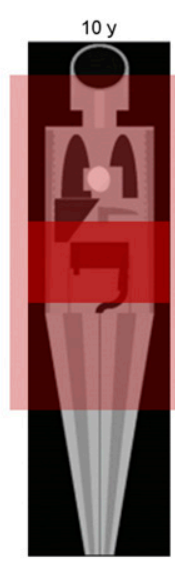

Scenario 4:

Abdomen Dx +

Whole Body AC

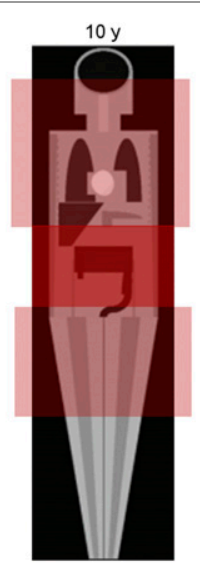

Scenario 5:

Abdomen Dx + Head and Chest AC

FIGURE 2. Visual representation of the 5 imaging scenarios for a $10-y$-old child (red $=\mathrm{Dx} C T$; pink = non-Dx CT). Scenario 1 is torso Dx CT in addition to torso non-Dx CT. Scenario 2 is torso Dx CT only. Scenario 3 is torso non-Dx CT only. Scenario 4 is Dx abdominal CT (from just above the diaphragm to just above the pelvis) in addition to torso non-Dx CT. Scenario 5 is multiseries CT with non-Dx in head/neck, chest, and legs while acquiring an abdominal Dx CT. Note that "whole body" in the figure is synonymous with "torso." 


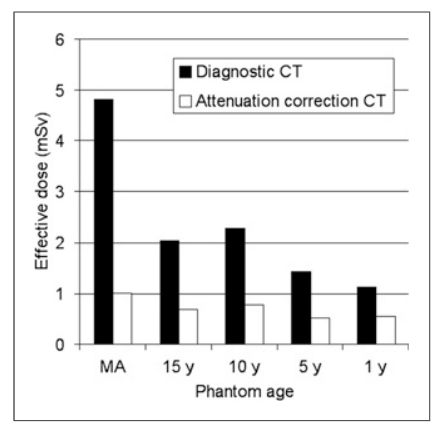

FIGURE 3. Attenuation correction (non-Dx) and Dx CT ED. ED estimates (in mSv) for Dx and non-Dx CT for various ages from a 1-y-old to a medium adult (MA). abdominal Dx. The ED estimate for each region was determined, and these values were summed for each phantom depending on the scenario. For instance, if the region of interest was the abdomen for a 10 -y-old and the patient was imaged according to our multiseries protocol (Fig. 2, scenario 5), the total ED was estimated by summing the non-Dx CT ED of the head/neck and chest with the Dx CT ED of the abdomen (0.018, 0.517, and $1.24 \mathrm{mSv}$, respectively).

Figure 3 shows the ED estimates for the non-Dx and Dx CT scans for different sizes of patient when using the Boston Children's Hospital PET/CT protocols. These ED estimates are for only the CT component of PET/CT and do not include the dose delivered by the radiopharmaceutical, typically $5.2-7.4 \mathrm{mSv}$ for ${ }^{18} \mathrm{~F}-\mathrm{FDG}$ according to the North American and EANM guidelines (30,31). Thus, CT AEC leads to a reasonable size-specific dose reduction. For both the non-Dx and the Dx CT scans, the 1- and-5-y-old patients received a lower radiation dose than larger patients and all the pediatric patients received a lower dose than the adult.

Figure 4 shows the ED estimates for a 10 -y-old for the 5 scenarios described. For the patient in scenario 5 receiving non-Dx CT of the head/neck and chest and standard-dose Dx CT of the abdomen, the ED estimate is $1.77 \mathrm{mSv}$, which is less than that received for scenario $1(3.09 \mathrm{mSv})$, scenario $2(2.30 \mathrm{mSv})$, or scenario $4(2.02 \mathrm{mSv})$. This demonstrates that merging the nonDx and Dx CT into a single multiseries acquisition can yield a reduction in radiation dose, that is a $44 \%, 23 \%$, and $12 \%$ reduction compared with scenarios 1,2 , and 4 .

\section{Case Study}

We present the case of a 13-y-old patient to illustrate one example of our multiseries protocol. This patient was diagnosed with lymphoblastic lymphoma after undergoing neck and chest Dx CT (Fig. 5A) and subsequent biopsy. To complete staging, torso ${ }^{18} \mathrm{~F}-\mathrm{FDG}$ PET/CT was performed, in addition to Dx CT of the abdomen/pelvis. Although the dose savings were predicted to be modest, we did not repeat the Dx CT of the neck and chest, as these regions had just been imaged at the time of the initial diagnosis. Using the multiseries PET/CT acquisition, non-Dx CT was performed in the neck and chest region, followed by Dx CT of the abdomen and pelvis. Additionally, non-Dx CT was obtained over the upper thighs for anatomic correlation for the final PET bed position (Fig. 5B). The neck/chest non-Dx CT was more than adequate to complement the earlier Dx CT of the same region, for attenuation correction and anatomic localization of the PET. The value of having Dx abdomen/pelvis CT is demonstrated by the ${ }^{18} \mathrm{~F}$ FDG-avid right renal lesion with low ${ }^{18} \mathrm{~F}-\mathrm{FDG}$ uptake, difficult to distinguish from renal clearance of ${ }^{18} \mathrm{~F}$-FDG without the coregistered contrast-enhanced Dx CT showing a low-attenuation lesion with corresponding ${ }^{18} \mathrm{~F}$-FDG uptake. This metastatic lesion would not have been apparent on a conventional noncontrast non-Dx CT. The regions that received non-Dx CT, the neck/chest $(0.3 \mathrm{mSv})$ as well as the legs $(0.3 \mathrm{mSv})$, received a considerably lower radiation dose than the abdomen/pelvis region $(3.4 \mathrm{mSv})$ where the standard Dx CT was performed (Fig. 5B). Overall, the implementation of our multiseries CT protocol provided valuable staging information to clinicians while lowering the patient's dose and saving the patient time.

\section{DISCUSSION}

PET/CT in children has been established as an essential imaging modality, particularly for oncology and neurology (1-7). However, since both the radiopharmaceutical and the CT components involve ionizing radiation, there is interest in keeping the radiation dose low while still providing high-quality Dx information to the clinician (3238 ). Although standards do exist for the use of ${ }^{18} \mathrm{~F}-\mathrm{FDG}$ in children $(30,39,40)$, this is not the case for the CT component. The survey results reported here indicated that there is considerable variation in the acquisition of the CT component even among dedicated pediatric institutions, which further shows the potential need for standardization. Over the past decade, several groups have reported on the optimization of pediatric PET/CT $(25,41-44)$. We have presented the Boston Children's approach as an option that uses AEC to manage radiation dose without setting specific levels for different-sized patients. Using the Boston Children's Hospital acquisition protocols, the non-Dx CT clearly led to a lower patient radiation dose. However, the relative magnitude of the difference varied with patient size (Fig. 4). For the adult, the non-Dx dose was about $20 \%$ of the Dx dose, whereas it was almost $50 \%$ for the 1 -y-old.

Incorporating Dx CT into the multiseries acquisition provided the lowest dose for the comparable Dx examinations. Although the dose savings is relatively small, this scenario is more efficient by providing both the nonDx and the Dx CT in a single acquisition. There will certainly be cases where the acquisition of a separate limited Dx CT will be more appropriate. For example, the ability to acquire a Dx chest CT with a breath hold and arms above the head may add significant value in patients with parenchymal lung lesions or notable findings in the lower lung near the diaphragm. In such cases, the separate Dx chest CT coupled with the nonDx torso CT is the best scenario and delivers only a slightly higher dose than the multiseries 


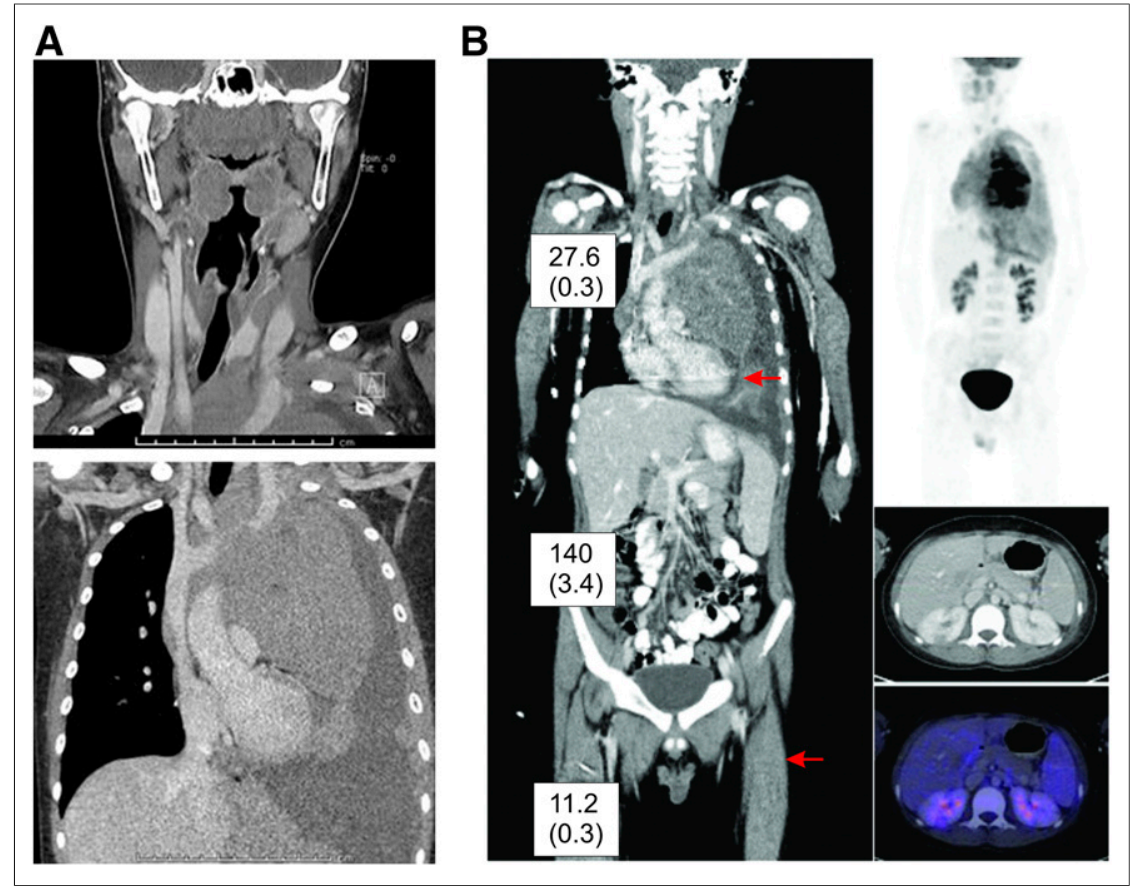

FIGURE 5. (A) Dx head/neck (top) and chest (bottom) CT (DLP = 139 mGy-cm; estimated ED = 2.2 $\mathrm{mSv}$ ) acquired before the PET/CT scan. (B) Multiseries PET/CT. Left panel shows the composite coronal CT image generated from the multiseries CT acquisition performed as part of the PET/CT acquisition. The head/neck and chest portions were acquired as non-Dx CT, the abdomen as Dx CT, and upper legs as non-Dx. The red arrows indicate transition between the different series, and different noise levels reflecting different CT techniques. The values in the 3 boxes are DLP (mGy-cm) and, in parentheses, the estimated ED (mSv) for each phase of the CT scan. Upper right panel is coronal image from resulting PET using the multiseries CT for attenuation correction. No transition in quality is discernable. Lower right panels show transverse CT (top) and fused PET/CT (bottom) demonstrating a low-attenuation lesion in the posterior right kidney and the corresponding ${ }^{18} \mathrm{~F}-\mathrm{FDG}$ uptake associated with the lesion. These latter findings could not have been made using noncontrast non-Dx CT.

Most Dx CT imaging requires intravenous contrast, and our survey revealed that only 2 of the 19 survey sites use intravenous contrast during their PET/CT examination. This may be due to concerns about iodinated contrast agents affecting tissue Hounsfield unit density and thereby compromising attenuation correction and accuracy of SUV measurements. Previous studies have shown little effect of properly administered contrast agents on SUV calculations ( $\sim 5 \%$ difference when comparing $\mathrm{SUV}_{\text {max }}$ and $\mathrm{SUV}_{\text {mean }}$ calculated from PET data corrected with both intravenous contrast present and absent attenuation-corrected CT data), which has been our experience as well $(45,46)$.

\section{CONCLUSION}

A multiseries PET/CT acquisition offers opportunities for dose reduction and examination optimization. As more hybrid imaging studies are performed and more imaging professionals with training in both radiology and nuclear medicine become aware of the multiseries PET/CT approach, the strategies we have outlined here will hopefully contribute to a broader discussion regarding standardization of CT techniques in the setting of pediatric $\mathrm{PET} / \mathrm{CT}$.

\section{ACKNOWLEDGMENTS}

We would like to acknowledge the individuals that contributed to our survey:

scenario (2.0 compared with $1.8 \mathrm{mSv}$ in our phantom study). Comparing the Boston Children's protocol to other centers, 5 of the 15 sites performing Dx CT acquired the CT in a manner similar to our traditional approach-that is, a limited CT scan acquired separately from the PET/CT scan but on the same scanner-whereas 6 sites used a multiseries protocol, similar to our new approach. Four of the 15 sites acquired the Dx CT over the entire PET field of view.

In pediatric imaging, the hope is to acquire the necessary imaging as efficiently as possible and with the lowest dose necessary while providing the clinician with the Dx information necessary for the patient's proper care. Most children are not imaged at dedicated pediatric centers, and thus centers that image children less frequently can benefit from guidance provided by the pediatric PET imaging community. We present our approach at Boston Children's Hospital as a reasonable method for pediatric PET/CT as it is simple and provides excellent image quality while maintaining the radiation dose at a level substantially lower than that for adults. However, a limitation in this discussion is that this approach may not be readily available with all PET/CT systems. In these cases, non-Dx PET/CT followed by Dx PET/CT limited to the area of clinical interest is a reasonable alternative. Our method for estimating the radiation dose from phantom experiments might also be a limitation, and so we are planning to obtain more accurate estimates through the use of realistic computerized phantoms and Monte Carlo simulations.
Christina Dodge of Texas Children's Hospital, Samuel Brady of St Jude Children's Research Hospital, Andrew Trout of Cincinnati Children's Hospital Medical Center, Jill Rothley of University of Michigan CS Mott Children's Hospital, Hollie A. Lai of Children's Hospital Los Angeles, Vinh Nguyen of Lucile Packard Children's Hospital Stanford, Dmitry Beyder of Barnes-Jewish and Saint Louis Children's Hospitals, Helen Nadel of British Columbia Children's Hospital, Timothy Turkington of Duke University, Michael Czachowski of Children's Hospital of Pittsburgh, Jennifer Foley of Children's Mercy Kansas City, Sarah McKenney of Children's National Health System, Ishtiaq Bercha of Children's Hospital Colorado, Nicholas Shkumat of The Hospital for Sick Children in Toronto, Lisa Aldape of Seattle Children's Hospital, Jon Adams of Massachusetts General Hospital, Nicholas Rupert of Ann \& Robert H. Lurie Children's Hospital of Chicago, and Kevin Edwards of Children's Hospital of Philadelphia.

\section{REFERENCES}

1. Treves ST. Pediatric Nuclear Medicine and Molecular Imaging. 4th ed. New York, NY: Springer; 2014.

2. Kiratli PÖ, Tuncel M, Bar-Sever Z. Nuclear medicine in pediatric and adolescent tumors. Semin Nucl Med. 2016;46:308-323.

3. Uslu L, Donig J, Link M, Rosenberg J, Quon A, Daldrup-Link HE. Value of ${ }^{18} \mathrm{~F}$ FDG PET and PET/CT for evaluation of pediatric malignancies. J Nucl Med. 2015;56:274-286. 
4. Kamal NM, Elsaban K. Role of ${ }^{18}$ F-FDG-PET/CT in assessment of pediatric Hodgkin's lymphoma. Q J Nucl Med Mol Imaging. April 1, 2014 [Epub ahead of print].

5. Harrison DJ, Parisi MT, Shulkin BL. The role of ${ }^{18} \mathrm{~F}-\mathrm{FDG}-\mathrm{PET} / \mathrm{CT}$ in pediatric sarcoma. Semin Nucl Med. 2017;47:229-241.

6. Raciborska A, Bilska K, Drabko K, et al. Response to chemotherapy estimates by FDG PET is an important prognostic factor in patients with Ewing sarcoma. Clin Transl Oncol. 2016;18:189-195.

7. Nguyen BD, Roarke MC, Chivers SF. Multifocal Langerhans cell histiocytosis with infiltrative pelvic lesions: PET/CT imaging. Clin Nucl Med. 2010;35:824-826.

8. Berrington de Gonzalez A, Journy N, Lee C, et al. No association between radiation dose from pediatric CT scans and risk of subsequent Hodgkin lymphoma. Cancer Epidemiol Biomarkers Prev. 2017;26:804-806.

9. Berrington de Gonzalez A, Salotti JA, McHugh K, et al. Relationship between paediatric CT scans and subsequent risk of leukaemia and brain tumours: assessment of the impact of underlying conditions. Br J Cancer. 2016;114:388-394.

10. Journy N, Ancelet S, Rehel JL, et al. Predicted cancer risks induced by computed tomography examinations during childhood, by a quantitative risk assessment approach. Radiat Environ Biophys. 2014;53:39-54.

11. Journy NM, Lee C, Harbron RW, McHugh K, Pearce MS, Berrington de Gonzalez A. Projected cancer risks potentially related to past, current, and future practices in paediatric CT in the United Kingdom, 1990-2020. Br J Cancer. 2017;116:109-116.

12. Kutanzi KR, Lumen A, Koturbash I, Miousse IR. Pediatric exposures to ionizing radiation: carcinogenic considerations. Int J Environ Res Public Health. 2016;13: E1057.

13. Miglioretti DL, Johnson E, Williams A, et al. The use of computed tomography in pediatrics and the associated radiation exposure and estimated cancer risk. JAMA Pediatr. 2013;167:700-707.

14. Siegel JA, Pennington CW, Sacks B. Subjecting radiologic imaging to the linear no-threshold hypothesis: a non sequitur of non-trivial proportion. J Nucl Med. 2017;58:1-6.

15. Siegel JA, Sacks B, Pennington CW, Welsh JS. Dose optimization to minimize radiation risk for children undergoing $\mathrm{CT}$ and nuclear medicine imaging is misguided and detrimental. J Nucl Med. 2017;58:865-868.

16. Fahey FH, Treves ST, Adelstein SJ. Minimizing and communicating radiation risk in pediatric nuclear medicine. J Nucl Med. 2011;52:1240-1251.

17. Health risks from exposure to low levels of ionizing radiation: BEIR VII phase II. Washington, DC: National Academic Press; 2006.

18. Brady SL, Shulkin BL. Ultralow dose computed tomography attenuation correction for pediatric PET CT using adaptive statistical iterative reconstruction. Med Phys. 2015;42:558-566.

19. Lee C, Pearce MS, Salotti JA, et al. Reduction in radiation doses from paediatric CT scans in Great Britain. Br J Radiol. 2016;89:20150305.

20. Fahey F, Treves ST, Lassmann M. Dose optimization in pediatric nuclear medicine. Clin Transl Imaging. 2016;4:5-11.

21. Fahey FH. Dosimetry of pediatric PET/CT. J Nucl Med. 2009;50:1483-1491.

22. Bushberg JT, Boone JM. The Essential Physics of Medical Imaging: Philadelphia, PA: Lippincott Williams \& Wilkins; 2011.

23. Boone J, Strauss K, Cody D, et al. AAPM report no. 204: size-specific dose estimates (SSDE) in pediatric and adult body CT examinations. College Park, MD: American Association of Physicists in Medicine; 2011.

24. Brady SL, Mirro AE, Moore BM, Kaufman RA. How to appropriately calculate effective dose for CT using either size-specific dose estimates or dose-length product. AJR. 2015;204:953-958.

25. Fahey FH, Palmer MR, Strauss KJ, Zimmerman RE, Badawi RD, Treves ST. Dosimetry and adequacy of CT-based attenuation correction for pediatric PET: phantom study 1. Radiology. 2007;243:96-104.
26. Rui X, Cheng L, Long Y, et al. Ultra-low dose CT attenuation correction for PET/CT: analysis of sparse view data acquisition and reconstruction algorithms. Phys Med Biol. 2015;60:7437-7460.

27. Lonn A. Evaluation of method to minimize the effect of x-ray contrast in PETCT attenuation correction [abstract]. IEEE Medical Imaging Conference. 2003; M6:146.

28. Harris PA, Taylor R, Thielke R, Payne J, Gonzalez N, Conde JG. Research electronic data capture (REDCap) — a metadata-driven methodology and workflow process for providing translational research informatics support. J Biomed Inform. 2009;42:377-381.

29. Deak PD, Smal Y, Kalender WA. Multisection CT protocols: sex-and age-specific conversion factors used to determine effective dose from dose-length product. Radiology. 2010;257:158-166.

30. Treves ST, Gelfand MJ, Fahey FH, Parisi MT. 2016 update of the North American consensus guidelines for pediatric administered radiopharmaceutical activities. J Nucl Med. 2016;57(12):15N-18N.

31. Lassmann M, Treves ST. Pediatric radiopharmaceutical administration: harmonization of the 2007 EANM paediatric dosage card (version 1.5.2008) and the 2010 North American consensus guideline [comment]. Eur J Nucl Med Mol Imaging. 2014;41:1636.

32. Goske MJ, Applegate KE, Boylan J, et al. The Image Gently campaign: working together to change practice. AJR. 2008;190:273-274.

33. Frush DP, Goske MJ, Hernanz-Schulman M. Computed tomography and radiation exposure. $N$ Engl J Med. 2008;358:851, author reply 852-853.

34. Stauss J, Franzius C, Pfluger T, et al. Guidelines for ${ }^{18} \mathrm{~F}-\mathrm{FDG}$ PET and PET-CT imaging in paediatric oncology. Eur J Nucl Med Mol Imaging. 2008;35:1581-1588.

35. Frush DP. Radiation, CT, and children: the simple answer is... it's complicated. Radiology. 2009;252:4-6.

36. Strauss KJ, Butler PF, Goske MJ, Ritenour ER. Image gently: reducing radiation dose in pediatric computed tomography through collaboration. Med Phys. 2009; 36:5719-5720.

37. Strauss KJ, Goske MJ. Estimated pediatric radiation dose during CT. Pediatr Radiol. 2011;41(suppl 2):472-482.

38. Frush DP, Strauss KJ. Image Gently: getting it right. J Am Coll Radiol. 2017; $14: 575-576$

39. Gelfand MJ, Parisi MT, Treves ST. Pediatric radiopharmaceutical administered doses: 2010 North American consensus guidelines. J Nucl Med. 2011;52:318322 .

40. Lassmann M, Treves ST, EANM/SNMMI Paediatric Dosage Harmonization Working Group. Paediatric radiopharmaceutical administration: harmonization of the 2007 EANM paediatric dosage card (version 1.5. 2008) and the 2010 North American consensus guidelines. Eur J Nucl Med Mol Imaging. 2014;41:1036-1041.

41. Alessio AM, Kinahan PE, Manchanda V, Ghioni V, Aldape L, Parisi MT. Weight-based, low-dose pediatric whole-body PET/CT protocols. J Nucl Med. 2009;50:1570-1577.

42. Gelfand MJ. Dosimetry of FDG PET/CT and other molecular imaging applications in pediatric patients. Pediatr Radiol. 2009;39(suppl 1):S46-S56.

43. Yang CC, Liu SH, Mok GS, Wu TH. Evaluation of radiation dose and image quality of CT scan for whole-body pediatric PET/CT: a phantom study. Med Phys. 2014;41:092505.

44. Parisi MT, Bermo MS, Alessio AM, Sharp SE, Gelfand MJ, Shulkin BL. Optimization of pediatric PET/CT. Semin Nucl Med. 2017;47:258-274.

45. Berthelsen AK, Holm S, Loft A, Klausen TL, Andersen F, Hojgaard L. PET/CT with intravenous contrast can be used for PET attenuation correction in cancer patients. Eur J Nucl Med Mol Imaging. 2005;32:1167-1175.

46. Aschoff P, Plathow C, Beyer T, et al. Multiphase contrast-enhanced CT with highly concentrated contrast agent can be used for PET attenuation correction in integrated PET/CT imaging. Eur J Nucl Med Mol Imaging. 2012;39:316-325. 